\title{
Association of STAT4 gene rs7574865G > T polymorphism with ulcerative colitis risk: evidence from 1532 cases and 3786 controls
}

Ling Xu, Wei-Qi Dai, Fan Wang, Lei He, Ying-Qun Zhou, Jie Lu, Xuan-Fu Xu, Chuan-Yong Guo

Department of Gastroenterology, Tenth People's Hospital of Tongji University, Shanghai, China

Submitted: 15 March 2012

Accepted: 11 May 2012

Arch Med Sci 2014; 10, 3: 419-424

DOI: 10.5114 /aoms.2014.43735

Copyright @ 2014 Termedia \& Banach

\section{Abstract}

Introduction: Several studies have reported the relationship between the STAT4 rs7574865G > T polymorphism as a susceptibility factor to ulcerative colitis (UC). However, the results have been controversial. Therefore, we conducted this meta-analysis to obtain the most reliable estimate of the association.

Material and methods: PubMed, Embase and Web of Science databases were searched. Crude odds ratios (OR) with $95 \%$ confidence intervals (CI) were extracted and pooled to assess the strength of the association between the STAT4 rs7574865G > T polymorphism and risk of UC. A total of five eligible studies including 1532 cases and 3786 controls based on the search criteria were involved in this meta-analysis.

Results: We observed that the STAT4 rs7574865G > T polymorphism was significantly correlated with UC risk when all studies were pooled into the meta-analysis (the allele contrast model: $O R=1.13,95 \% \mathrm{Cl}=1.02-1.25$; the heterozygote codominant model: $\mathrm{OR}=1.22,95 \% \mathrm{Cl}=1.04-1.43$; the dominant model: $O R=1.25,95 \% \mathrm{Cl}=1.07-1.45)$. In the stratified analysis by ethnicity, significant associations were observed in Spanish for the allele contrast model $(\mathrm{OR}=1.20 ; 95 \% \mathrm{Cl}=1.04-1.39)$, for the homozygote codominant model $(\mathrm{OR}=1.57 ; 95 \% \mathrm{Cl}=1.07-2.31)$, for the dominant model $(\mathrm{OR}=1.20 ; 95 \% \mathrm{Cl}=1.01-1.43)$, and for the recessive model $(\mathrm{OR}=1.50$; $95 \% \mathrm{Cl}=1.03-2.19)$.

Conclusions: This meta-analysis suggests that the STAT4 rs7574865G > T polymorphism is a low-penetrant risk factor for UC, especially in Spanish.

Key words: STAT4, ulcerative colitis, genetic polymorphisms, mutation risk.

\section{Introduction}

Inflammatory bowel disease (IBD), including Crohn's disease (CD) and ulcerative colitis (UC), is a common health problem worldwide. It results from an interaction between genetic, environmental, inflectional, and other factors [1]. Preliminary findings suggest that genetic susceptibility plays a large part in the predisposition to UC [2,3], and even the rare cases with Takayasu arteritis may display varietal genetic susceptibility $[4,5]$.

The signal transducer and activator of transcription-4 (STAT4) gene was found to be associated with multiple autoimmune diseases, such

\author{
Corresponding author: \\ Dr. Chuan-Yong Guo \\ Department \\ of Gastroenterology \\ Tenth People's Hospital \\ of Tongji University \\ Shanghai 200072, China \\ Phone: +86-21-66302535 \\ Fax: +86-21-66303983 \\ E-mail: guochuanyong@ \\ hotmail.com
}


as systemic lupus erythematosus, rheumatoid arthritis, type 1 diabetes, Wegener's granulomatosis, and ulcerative colitis [6]. Mutated allele T of the STAT4 rs7574865 SNP plays a key role in these autoimmune diseases $[7,8]$. More recently, several studies have assessed the relationship between the polymorphism of STAT4 $G>T$ and the susceptibility to $U C$, but the results have been controversial [9-12]. Therefore, we conducted this meta-analysis to confirm these associations.

\section{Material and methods}

\section{Identification and eligibility of relevant studies}

The search terms "STAT4", "ulcerative colitis", "genotype", "polymorphism" and "variant" were employed to explore publications in PubMed, ISI Web of Knowledge and Embase databases for relevant reports (last search update October 2011). Only studies published in the English language were included. We did not define any minimum number of patients to be included for meta-analysis. When multiple studies of the same patient population were identified, we included the published report with the largest sample size (Table I).

\section{Inclusion and exclusion criteria}

The following inclusion criteria were used to select literature for this analysis: (a) evaluation of the STAT4 rs7574865G > T polymorphism and UC, (b) only case-control studies were considered, (c) sufficient published data to estimate an odds ratio (OR) with 95\% confidence interval (CI). Major exclusion criteria were: (1) no control population, (2) no available genotype frequency, and (3) duplicated studies.

\section{Data extraction}

For each study, the following data were collected: first author's surname, year of publication, country of origin, ethnicity, criteria of enrolled patients, genotyping method, total numbers of cases and controls as well as numbers of cases and controls with GG, GT and TT genotypes. The strength of the association between STAT4 rs7574865G > T polymorphism and UC risk was estimated using $\mathrm{OR}$, with the corresponding $95 \% \mathrm{Cl}$. Disagreement was resolved by discussion until a consensus was reached between the two authors. We did not define any minimum number of patients for inclusion in our meta-analysis.

\section{Statistical analysis}

The risk of UC associated with STAT4 rs7574865G > T was estimated for each study by OR with $95 \% \mathrm{Cl}$. For five studies, we analysed the relationship for the allele contrast model ( $T$ vs. G). At the same time, due to lack the specific genotypes of Glas's literature reported by Glas, we estimated the association under other four different types of OR, namely the homozygote codominant model (TT vs. GG), the heterozygote codominant model (GT vs. GG), the dominant model (TT+GT vs. GG) and the recessive model (TT vs. GT+GG). Hardy-Weinberg equilibrium (HWE) was tested by the $\chi^{2}$ test. The Q-statistic was used to investigate the degree of heterogeneity between the trials, and a $p$-value of 0.10 for the Q-test indicated a lack of heterogeneity among studies. We used the fixed-effects model and the random-effects model based on the Mantel-Haenszel method [13] and the DerSimonian and Laird method [14], respectively, to combine values from each of the studies. A sensitivity analysis was also performed by omitting each study in turn to identify potential outliers. All of the statistical analyses were performed with Review Manage version 4.3 and STATA version 12.0 using two-sided $p$-values.

\section{Results}

\section{Study characteristics}

We obtained 12 studies about the association between STAT4 rs7574865 polymorphism. Following the above inclusion and exclusion criteria, 5 publications were included in the final metaanalysis [9-12]. Characteristics of studies focusing on STAT4 rs7574865G > T are summarized in Tablell. Genotypes and separate $p$ values for STAT4 rs7574865 polymorphism are listed in Table III.

\section{Meta-analysis results}

Table III lists the main results of pooled ORs for STAT4 rs7574865G > T polymorphism and UC risk. We observed that the STAT4 rs7574865G > T polymorphism was significantly correlated with UC risk when all studies were pooled into the meta-analysis (the allele contrast model: $\mathrm{OR}=1.13$, $95 \% \mathrm{Cl}=1.02-1.25, p=0.224$ for heterogeneity, Figure 1; the heterozygote codominant model: $\mathrm{OR}=1.22,95 \% \mathrm{Cl}=1.04-1.43, p=0.49$ for heterogeneity, Figure 2; the dominant model: $\mathrm{OR}=$ $1.25,95 \% \mathrm{Cl}=1.07-1.45, p=0.54$ for heterogeneity, Figure 3 ). In the analysis stratified by ethnicity, significant associations were observed in Spanish for the allele contrast model $(\mathrm{OR}=1.20 ; 95 \% \mathrm{Cl}$ $=1.04-1.39 ; p=0.42$ for heterogeneity), for the homozygote codominant model $(\mathrm{OR}=1.57$; $95 \% \mathrm{Cl}=1.07-2.31 ; p=0.69$ for heterogeneity), for the dominant model $(\mathrm{OR}=1.20 ; 95 \% \mathrm{Cl}=$ $1.01-1.43 ; p=0.40$ for heterogeneity), and for the recessive model $(\mathrm{OR}=1.50 ; 95 \% \mathrm{Cl}=1.03-2.19$; $p=0.78$ for heterogeneity). 
Table I. Characteristics of UC studies included in the meta-analysis

\begin{tabular}{|lcccccc|}
\hline First author/published year & $\begin{array}{c}\text { Study population } \\
\text { of cases }\end{array}$ & Method & Source & Cases & Controls & $\begin{array}{c}\text { HWE } \\
\text { of controls }\end{array}$ \\
\hline Bouzid [9] 2009 & North Africa & $\begin{array}{c}\text { SNP genotyping } \\
\text { assays }\end{array}$ & HB & 68 & 162 & 0.494 \\
\hline Diaz-Gallo [8] 2010 & Spanish & $\begin{array}{c}\text { SNP genotyping } \\
\text { assays }\end{array}$ & HB & 402 & 1296 & 0.888 \\
\hline Moon [7] 2010 & Korean & $\begin{array}{c}\text { SNP genotyping } \\
\text { assays }\end{array}$ & HB & 246 & 229 & 0.934 \\
\hline Martinez [10] 2008 & Spanish & $\begin{array}{c}\text { SNP genotyping } \\
\text { assays }\end{array}$ & HB & 352 & 716 & 0.968 \\
\hline Glas [11] 2010 & Caucasian & SNP genotyping \\
assays & HB & 464 & 1383 & 0.215 \\
\hline
\end{tabular}

Table II. Genotypes and $p$-values of STAT4 rs7574865G > T polymorphism included in the meta analysis

\begin{tabular}{|lcccccccccc|}
\hline First author & \multicolumn{4}{c}{ Cases } & \multicolumn{7}{c|}{ Controls } \\
\cline { 2 - 11 } & GG & GT & TT & G & T & GG & GT & TT & G & T \\
\hline Bouzid & 39 & 25 & 4 & 103 & 33 & 113 & 46 & 3 & 272 & 52 \\
\hline Diaz-Gallo & 241 & 137 & 24 & 619 & 185 & 813 & 428 & 55 & 2054 & 538 \\
\hline Moon & 97 & 131 & 18 & 325 & 167 & 106 & 100 & 23 & 312 & 146 \\
\hline Martinez & 209 & 123 & 20 & 541 & 163 & 470 & 220 & 26 & 1160 & 272 \\
\hline Glas & - & - & - & 729 & 199 & - & - & - & 2171 & 595 \\
\hline
\end{tabular}

Table III. Results of meta-analysis for STAT4 rs7574865 polymorphisms and UC risk

\begin{tabular}{|c|c|c|c|c|c|c|}
\hline \multirow[t]{2}{*}{ Study groups } & \multicolumn{2}{|c|}{ Allele contrast model } & \multicolumn{3}{|c|}{ Homozygote codominant } & \\
\hline & OR $(95 \% \mathrm{Cl})$ & Value of $p$ & \multicolumn{2}{|c|}{ OR $(95 \% \mathrm{Cl})$} & \multicolumn{2}{|l|}{ Value of $p$} \\
\hline Total & $1.13(1.02-1.25)$ & 0.006 & \multicolumn{2}{|c|}{$1.39(1.00-1.92)$} & 0.05 & \\
\hline \multicolumn{7}{|l|}{ Ethnicity } \\
\hline Spanish & $1.20(1.04-1.39)$ & 0.01 & \multicolumn{2}{|c|}{$1.57(1.07-2.31)$} & 0.02 & \\
\hline Korean & $1.10(0.84-1.44)$ & - & \multicolumn{2}{|c|}{$0.86(0.44-1.68)$} & - & \\
\hline North Africa & $1.33(0.82-2.15)$ & - & $3.86(0.83-18$ & & - & \\
\hline \multirow[t]{2}{*}{ Study groups } & \multicolumn{2}{|c|}{ Heterozygote codominant } & \multicolumn{2}{|c|}{ Dominant model } & \multicolumn{2}{|c|}{ Recessive model } \\
\hline & OR $(95 \% \mathrm{Cl})$ & $\begin{array}{l}\text { Value } \\
\text { of } p\end{array}$ & OR $(95 \% \mathrm{Cl})$ & $\begin{array}{c}\text { Value } \\
\text { of } p\end{array}$ & OR $(95 \% \mathrm{Cl})$ & $\begin{array}{l}\text { Value } \\
\text { of } p\end{array}$ \\
\hline Total & $1.22(1.04-1.43)$ & 0.01 & $1.25(1.07-1.45)$ & 0.004 & $1.30(0.82-2.08)$ & 0.26 \\
\hline \multicolumn{7}{|l|}{ Ethnicity } \\
\hline Spanish & $1.15(0.96-1.38)$ & 0.12 & $1.20(1.01-1.43)$ & 0.04 & $1.50(1.03-2.19)$ & 0.04 \\
\hline Korean & $1.43(0.98-2.09)$ & - & $1.32(0.92-1.91)$ & - & $0.71(0.37-1.35)$ & - \\
\hline North Africa & $1.57(0.86-2.89)$ & - & $1.71(0.95-3.08)$ & - & $3.31(0.72-15.22)$ & - \\
\hline
\end{tabular}

\section{Publication bias}

We performed Begg's funnel plot and Egger's test to assess the publication bias of the literature. The results did not show any evidence of publication bias in all the comparisons. We present funnel plot for ORs of C vs. T in Figure 4. Also, the results of Egger's test still did not suggest any evidence of publication bias ( $p=0.679$ for T vs. G; $p=0.134$ for GT vs. GG; $p=0.104$ for TC vs. TT).

\section{Discussion}

The Stat signalling pathways play important roles in carcinogenesis and immunopathology of many malignancies and immunologic diseases. 


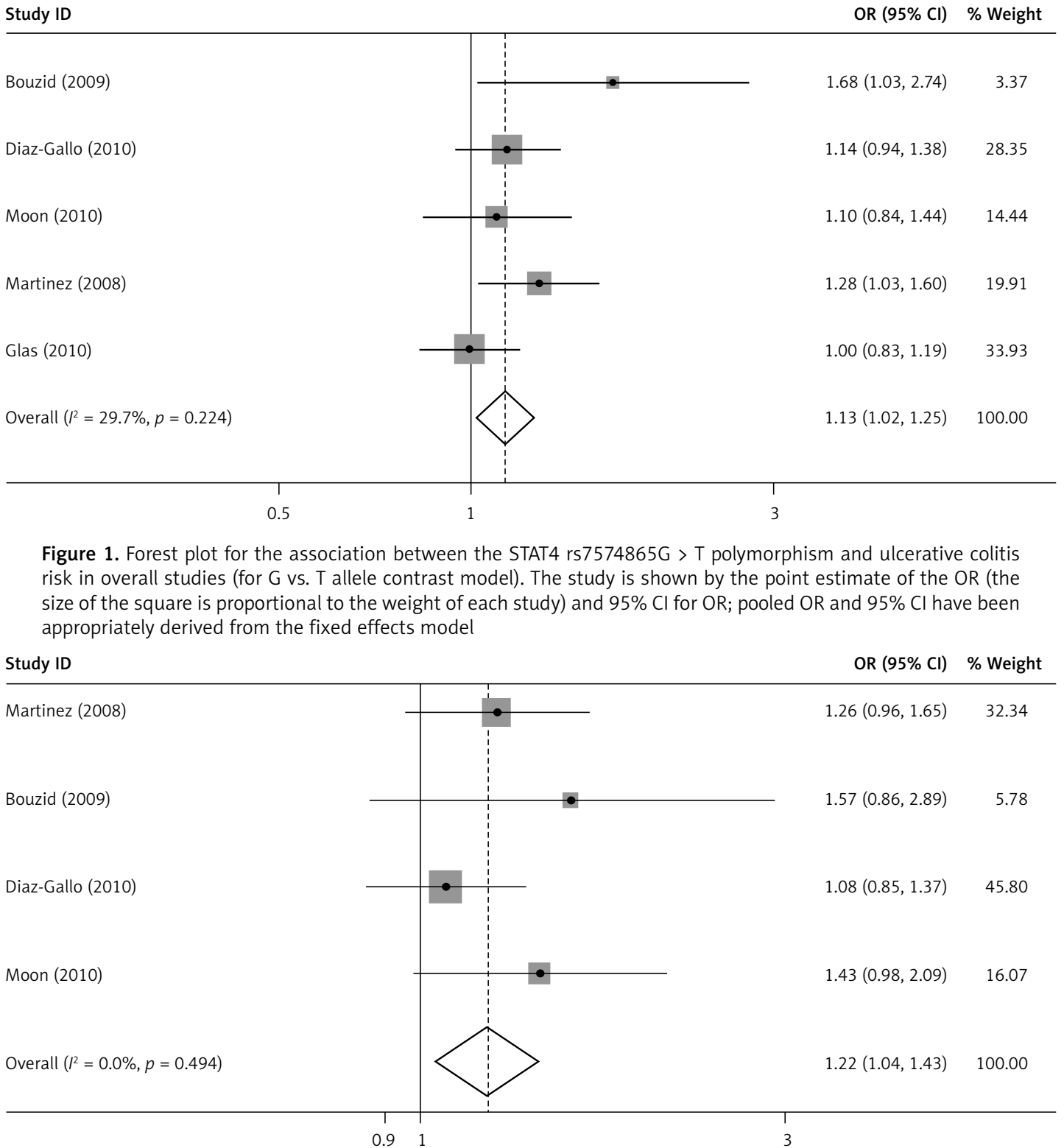

Figure 2. Forest plot for the association between the STAT4 rs7574865G > T polymorphism and ulcerative colitis risk in overall studies (for the heterozygote codominant model, fixed effects model)

Recent studies have implicated their members' possible involvement in the pathogenesis of IBD. For example, the expression and/or activation of interleukin 12 (IL-12) and Stat4, including phosphorylated Stat4, suggest that proinflammatory IL-12/Stat4 signalling or IL-23/Stat4 are likely candidate pathways involved in the inflammatory pathology in UC and colorectal cancer (CRC) [15-17]. Moreover, it is generally recognized that there is an increased risk of CRC in patients with UC, and the overall prevalence of manifest CRC in patients with UC is unacceptably high. So, this understanding may have an important impact on patients with certain risk factors, such as STAT4, etc [18]. Some researchers have examined the association of STAT4 rs7574865G > T polymorphism with UC risk and treatments. Based on previous studies reporting increased sensitivity to IFN- $\alpha$ in lupus patients carrying the risk variant of STAT4 might contribute to increased mucosal inflammation in IBD patients and to the response to immunosuppressive and immunomodulatory therapies [19], and a significant relationship was observed in several but not all studies [20,21].

The aim of our study was to demonstrate the role of STAT4 rs7574865G > T polymorphism in the relationship with UC risk using a meta-analysis. Interestingly, we observed that STAT4 rs7574865G $>\mathrm{T}$ was significantly correlated with UC risk and the T allele of the STAT4 rs7574865G > T variant was a low-penetrant risk factor for UC. Simultaneously there was evidence to indicate that STAT4 


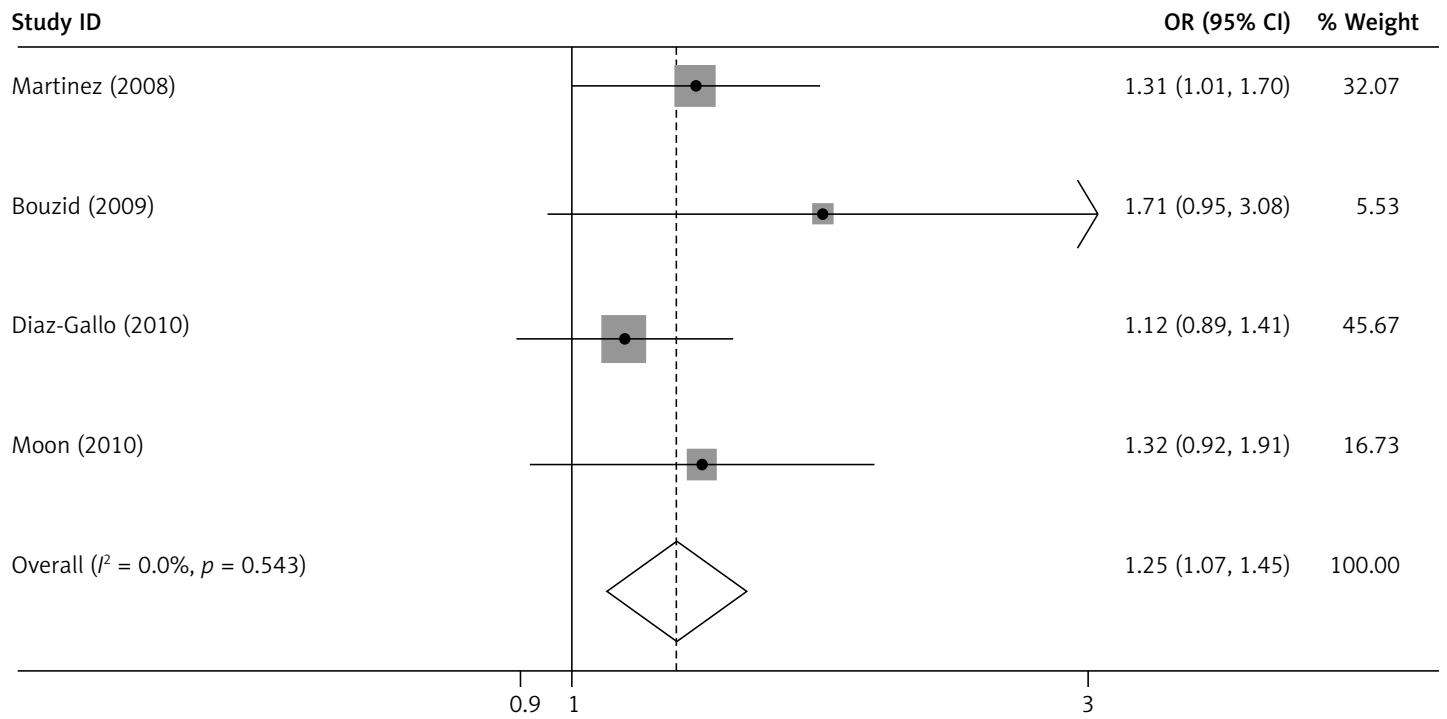

Figure 3. Forest plot for the association between the STAT4 rs7574865G > T polymorphism and ulcerative colitis risk in overall studies (for the dominant model, fixed effects model)

rs7574865G > T polymorphism was associated with increased risk of UC in Spanish and mixed ethnicities.

Of course, we must note that our study population was small, so it is still necessary to conduct larger sample studies considering gene-gene and gene-environment interactions, and using standardized unbiased genotyping methods, homogeneous UC patients, and sufficiently matched controls. In addition, we also should consider the relationship between treatment response and STAT4 polymorphisms in various types of UC in future work for a comprehensive evaluation of the relevance of this polymorphism for UC clinical characteristics, for example, whether it is similar to TLR2 polymorphisms that can predict the incidence of steroid-dependent UC and the response to azathioprine treatment, etc [12, 22, 23].

Recently, genome-wide association studies (GWAS) have helped scientists understand the inheritance patterns of disorders on a global scale. In particular, this may lead to an enormous boost to the identification of susceptibility genes for several diseases, including inflammatory bowel disease, but there are some insurmountable problems, the most critical being that some high-risk sites for low frequency are masked by low-risk sites for high frequency, so this may result in the analysis not being comprehensive, with the loss of a lot of meaningful SNP sites. For example, Rivas et al. have identified more than 1,000 susceptibility loci for IBD through GWAS; further analysis found that only four additional independent risk factors, NOD2, two additional protective variants in IL23R, and a protective splice variant in CARD9, are associated with IBD [21], while some of the classic sites, such as HLA, cytokine and STAT4 gene polymorphisms [24, 25], cannot be identified.

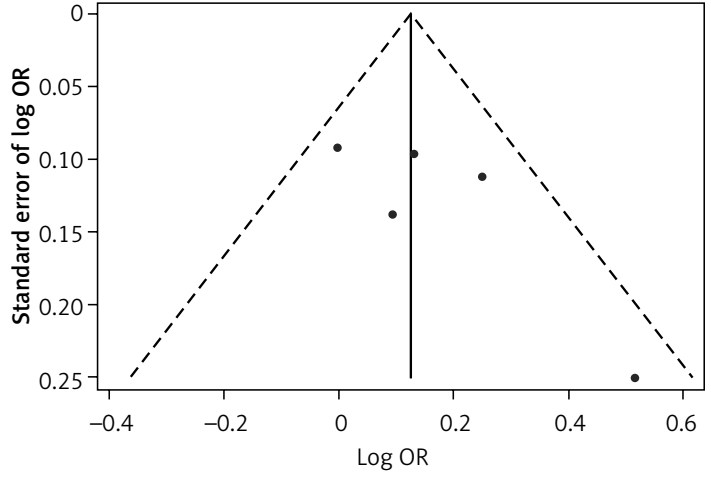

Figure 4. Funnel plot analysis for odds ratios of $G$ allele compared with $T$ allele overall

In conclusions, our study shows a genetic association between STAT4 rs7574865G > T variant and increased susceptibility to UC. However, it needs to be further evaluated in larger sample collections in diverse ethnic populations and more types of UC along with clinical characteristics, to confirm our findings.

\section{Acknowledgments}

Wei-Qi Dai and Ying-Qun Zhou contributed equally to this work.

This research was supported in part by grants from the Biomedical Project Science Technology Committee of Shanghai (10149018001).

\section{References}

1. Benchimol El, Fortinsky KJ, Gozdyra P, et al. Epidemiology of pediatric inflammatory bowel disease: a systematic review of international trends. Inflamm Bowel Dis 2011; 17: 423-39.

2. Thompson Al, Lees CW. Genetics of ulcerative colitis. Inflamm Bowel Dis 2011; 17: 831-48. 
3. Cho JH. The genetics and immunopathogenesis of inflammatory bowel disease. Nat Rev Immunol 2008; 8: 458-66.

4. Takahashi N, Tanabe K, Sugamori T, et al. Association between Takayasu arteritis and ulcerative colitis - case report and review of serological HLA analysis. Med Sci Monit 2011; 17: CS81-84.

5. Horai Y, Miyamura T, Shimada K, et al. A case of Takayasu's arteritis associated with human leukocyte antigen A24 and B52 following resolution of ulcerative colitis and subacute thyroiditis. Intern Med 2011; 50: 151-4.

6. Thompson Al, Lees CW. Genetics of ulcerative colitis. Inflamm Bowel Dis 2011; 17: 831-48.

7. Korman BD, Kastner DL, Gregersen PK, et al. STAT4: genetics, mechanisms, and implications for autoimmunity. Curr Allergy Asthma Rep 2008; 8: 398-403.

8. Glas J, Seiderer J, Nagy M, et al. Evidence for STAT4 as a common autoimmune gene: rs7574865 is associated with colonic Crohn's disease and early disease onset. PLoS One 2010; 5: e10373.

9. Moon CM, Cheon JH, Kim SW, et al. Association of signal transducer and activator of transcription 4 genetic variants with extra-intestinal manifestations in inflammatory bowel disease. Life Sci 2010; 86: 661-7.

10. Diaz-Gallo LM, Palomino-Morales RJ, Gómez-García M, et al. STAT4 gene influences genetic predisposition to ulcerative colitis but not Crohn's disease in the Spanish population: a replication study. Hum Immunol 2010; 71: 515-9.

11. Zervou MI, Goulielmos GN, Castro-Giner F, et al. STAT4 gene polymorphism is associated with psoriasis in the genetically homogeneous population of Crete, Greece. Hum Immunol 2009; 70: 738-41.

12. Martínez A, Varadé J, Márquez A, et al. Association of the STAT4 gene with increased susceptibility for some immune-mediated diseases. Arthritis Rheum 2008; 58: 2598-602.

13. Jose S, George PS, Mathew A. Assessment of confounding and interaction using the mantel-haenszel risk estimation method. Asian Pac J Cancer Prev 2008; 9: 323-5.

14. Kjellsson MC, Zingmark PH, Jonsson EN, et al. Comparison of proportional and differential odds models for mixed-effects analysis of categorical data. J Pharmacokinet Pharmac 2008; 35: 483-501.

15. Pang $\mathrm{YH}$, Zheng $\mathrm{CQ}$, Yang $\mathrm{XZ}$, et al. Increased expression and activation of IL-12-induced Stat4 signaling in the mucosa of ulcerative colitis patients. Cell Immunol 2007; 248: 115-20.

16. Lee YH, Woo JH, Choi SJ, et al. Association between the rs 7574865 polymorphism of STAT4 and rheumatoid arthritis: a meta-analysis. Rheumatol Int 2010; 30: 661-6.

17. Alarcón-Riquelme ME. Recent advances in the genetics of autoimmune diseases. Ann N Y Acad Sci 2007; 1110 1-9.

18. Yamaguchi N, Isomoto $\mathrm{H}$, Shikuwa $\mathrm{S}$, et al. Proximal extension of backwash ileitis in ulcerative-colitis - associated colon cancer. Med Sci Monit 2010; 16: CS87-91.

19. Kariuki SN, Kirou KA, MacDermott EJ, et al. Cutting edge: autoimmune discase risk variant of STAT4 confers increased sensitivity to IFN-alpha in lupus patients in vivo. J Immunol 2009; 182: 34-8.

20. Mudter J, Weigmann B, Bartsch B, et al. Activation pattern of signal transducers and activators of transcription (STAT) factors in inflammatory bowel diseases. Am J Gastroenterol 2005; 100: 64-72.

21. Rivas MA, Beaudoin M, Gardet A, et al. Deep resequencing of GWAS loci identifies independent rare variants associated with inflammatory bowel disease. Nat Genet 2011; 43: 1066-73.

22. Chebli LA, Felga GG, Chaves LD, et al. Early onset steroid-dependent ulcerative colitis is a predictor of Azathioprine response: a longitudinal 12-month follow-up study. Med Sci Monit 2010; 16: PI1-6.

23. Ehteshami-Afshar S, Nikfar S, Rezaie A, Abdollahi M. A systematic review and meta-analysis of the effects of infliximab on the rate of colectomy and post-operative complications in patients with inflammatory bowel disease. Arch Med Sci 2011; 7: 1000-12.

24. Sands BE. Inflammatory bowel disease: past, present, and future. J Gastroenterol 2007; 42: 16-25.

25. Peña AS. Genetics of inflammatory bowel disease. The candidate gene approach: susceptibility versus disease heterogeneity. Dig Dis 1998; 16: 356-63. 